\title{
Palliative esophagectomy in unexpected metastatic disease: sense or nonsense?
}

\author{
Lieven P Depypere $\mathbb{D}$, Johnny Moons, Toni E Lerut, \\ Willy Coosemans, Hans Van Veer $(\mathbb{D}$ and Philippe R Nafteux
}

@SAGE

\begin{abstract}
Background: Despite integrated positron emission tomography and computed tomography screening before and after neoadjuvant treatment in patients with locally advanced esophageal cancer, unexpected metastatic disease is still found in some patients during surgery. Should then esophagectomy be aborted or is there a place for palliative resection?

Methods: Between 2002 and 2015, 68I patients with potentially resectable esophageal cancer were sheduled for neoadjuvant therapy and subsequent esophagectomy. In 552 patients, a potentially curative esophagectomy was performed. In 12 patients, unexpected disease was discovered during surgery but esophagectomy was performed with synchronous resection of metastases; 10 of them had oligometastatic disease ( $\leqslant 4$ single-organ metastases). Esophagectomy was not performed in 117 patients (because of disease progression in 50); 14 were also single-organ oligometastatic. Data of 10 single-organ oligometastatic patients who underwent esophageal resection (group I) were compared those of 10 non-resected but treated counterparts (group 2) and with 228 patients who underwent potentially curative esophagectomy with persistent pathological lymph nodes (group 3).

Results: Five oligometastatic esophagectomy patients had lung metastases: I peritoneal, 2 adrenal, I pleural, and I pancreatic. Two oligometastatic non-resected patients had lung, 5 liver, and 3 brain metastases. Median overall survival was $21.4,12$. I, and 20.2 months in the respective groups (group I vs. group $2 p=0.042$; group 2 vs. group $3 p=0.002$; group I vs. group $3 p=0.88$ ).

Conclusions: Survival is longer in patients undergoing palliative esophagectomy with unexpected single-organ oligometastatic disease and comparable to survival in patients with persistent pathological lymph nodes. Palliative resection in these patients seems to be justified.
\end{abstract}

\section{Keywords}

Chemoradiotherapy, Esophageal neoplasms, Esophagectomy, Neoplasm metastasis, Treatment outcome, palliative treatment, oligometastatic disease

\section{Introduction}

Surgical treatment is the foundation of today's curative treatment for esophageal cancer. Current guidelines advocate the use of integrated 18-fluorodeoxyglucose positron-emission tomography (FDG-PET) and computed tomography (CT) for initial staging to exclude patients with organ metastases. ${ }^{1,2}$ Since recent randomized trials have shown better local tumor control and a survival benefit in patients undergoing neoadjuvant treatment, the majority of patients with potentially resectable esophageal cancer undergo neoadjuvant therapy followed by surgery. ${ }^{3,4}$ Several groups have recommended restaging with CT only or FDG-PET/CT after neoadjuvant treatment because futile surgery can be cost-effectively avoided in $5 \%$ to $10 \%$ of patients. ${ }^{5-8}$ Nevertheless, Hulshoff and colleagues ${ }^{5}$ described $4 \%$ false-negative findings after restaging with CT only, and Anderegg and colleagues ${ }^{8}$ found the same result after restaging with FDG-PET/CT, leading to metastases discovered intraoperatively. In patients with intraoperatively discovered synchronous metastases

Department of Thoracic Surgery, University Hospitals, Leuven, Belgium

Corresponding author:

Lieven P Depypere, Department of Thoracic Surgery, University Hospitals Leuven-Gasthuisberg, Herestraat 49, 3000 Leuven, Belgium. Email: lieven.depypere@uzleuven.be 
despite thorough clinical staging, the question arises of whether surgery is still considered futile and the procedure needs to be aborted, or if one can proceed with radical treatment of the primary tumor and metastases with a curative intent. Indeed, more and more cancer treatment programs, such as breast carcinoma, nonsmall-cell lung carcinoma, and colorectal carcinoma have oligometastatic protocols. ${ }^{9-11}$ Therefore, the aim of this study was to retrospectively review the outcome of radical treatment in patients with locally advanced esophageal cancer and unexpected intraoperatively discovered metastases.

\section{Patients and methods}

The research was conducted in accordance to the World Medical Association Declaration of Helsinki. The protocol of this study was approved by our institutional review board and ethical committee (S61493), and the need for written informed consent was waived, given the retrospective nature of the study. Between 2002 and 2015, 681 consecutive patients with locally advanced resectable esophageal cancer (cT1-4 N+ or cT3-4N0) were scheduled for neoadjuvant therapy in our hospital or a referring center, for esophageal resection in our department. All patients underwent at least an endoscopy with biopsy for histological proof of malignancy and FDG-PET/CT for further clinical staging. Endoscopic ultrasound for local clinical staging was performed in the majority of patients when possible (patency of the esophageal tumor). When indicated, clinical staging was completed by other examinations as bronchoscopy, endobronchial ultrasonography, ultrasonography, magnetic resonance imaging (whether or not combined with punctures), bone scintigraphy, mediastinoscopy, thoracoscopy and/or laparoscopy. All patients who completed neoadjuvant therapy underwent restaging by at least FDG-PET/CT 2-6 weeks after termination of neoadjuvant therapy. All patients were discussed at least once (before, during, or after neoadjuvant treatment) by the institutional multidisciplinary tumor board dedicated to esophageal cancer. All patients undergoing esophagectomy underwent a transthoracic esophagectomy with at least standard two-field lymph node dissection. The exact procedure depended on the patients general condition, tumor histology and location, as described previously by our group. ${ }^{12}$

As depicted in the patient flow chart (Figure 1), 552 patients underwent potentially curative esophagectomy. Twelve patients had intraoperatively discovered metastatic disease but had a subsequent esophagectomy. Esophagectomy was not performed in 117 patients for several reasons including disease progression identified during restaging imaging or an exploratory procedure in 50 cases.

The literature on surgery for oligometastatic disease in esophageal cancer, and particularly, its definition, is scarce. In non-small-cell lung cancer, there is a consensus that survival can be improved with radical ablative treatment when oligometastatic disease is defined as 3 to 5 metastases in a single organ. ${ }^{13}$ Therefore, we chose the current definition by consensus, based on nonsmall-cell lung cancer literature.

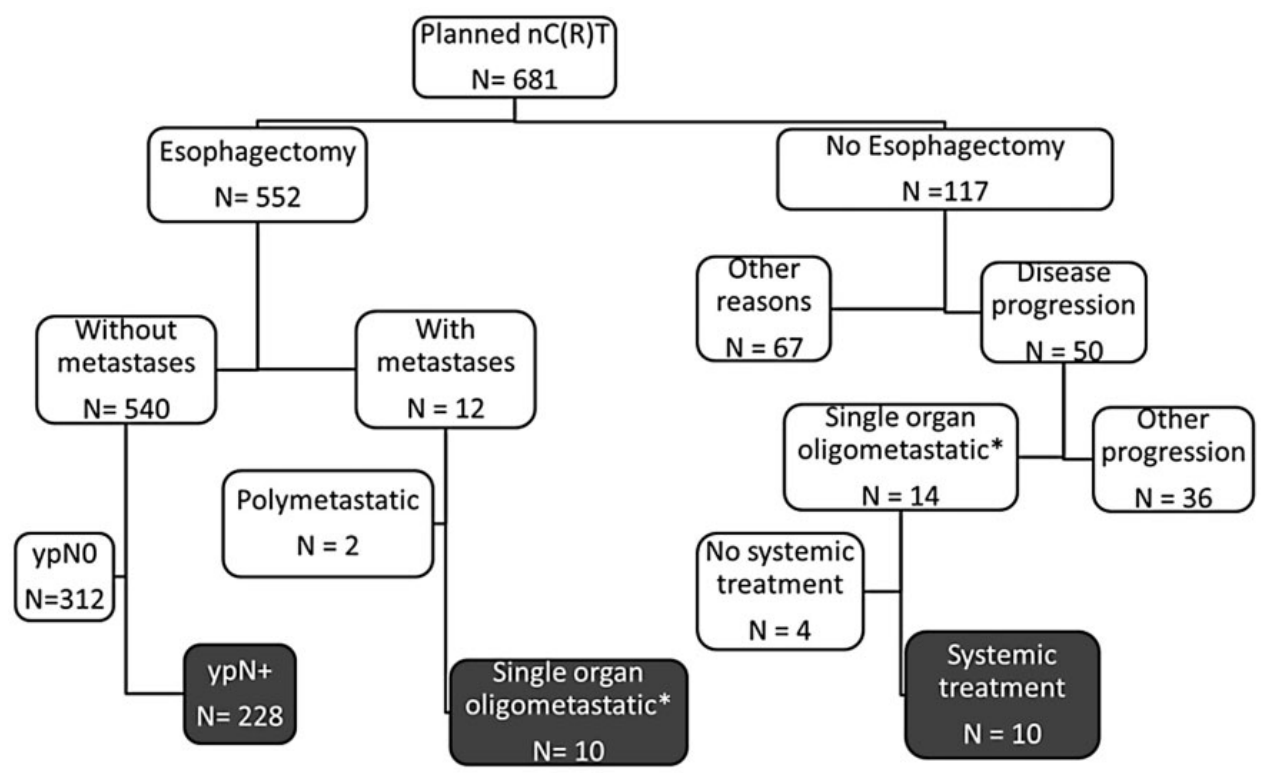

Figure I. The patient flow chart. *Four or less metastases in a single organ. nC(R)T: neoadjuvant chemo(radiation)therapy. 
Data of patients with intraoperatively discovered oligometastatic disease who underwent subsequent esophagectomy (group 1) were compared to those of patients who had oligometastatic progressive disease discovered during restaging after neoadjuvant therapy and who underwent maximal medical therapy for their disease (at least one line of systemic treatment and additional local treatment for locations beyond the bloodbrain barrier, group 2). Both groups were compared to group 3 that comprised patients treated with curative intent in whom persistent pathological lymph nodes were found after esophagectomy. In these 3 groups, patient-related, disease-related, and treatment-related characteristics were retrospectively analysed.

Summary statistics are presented as median and range for continuous variables and as tenths for categorical variables. These variables were compared between groups with unpaired $t$ tests. Patient survival was estimated using Kaplan-Meier curves from the date of histological diagnosis, and compared by log-rank tests. A $p$ value less than 0.05 was considered significant. All analyses were performed using IBM SPSS software version 24 (IBM Corp., Armonk, NY, USA).

\section{Results}

Ten of 12 patients with intraoperatively discovered metastatic disease who underwent subsequent esophagectomy were oligometastatic (group 1). All detectable metastases during surgery were excised. Two patients had a right-sided approach (McKeown) and 8 had a left thoracoabdominal approach. Only the lung at the side of surgical approach could be completely evaluated during surgery. Five patients had lung metastases (all had wedge resections: 3 in the left lower lobe, one in the left upper lobe, and one in the right upper lobe), 2 had adrenal metastases (in both cases, complete resection of the left adrenal gland was performed), 1 had peritoneal (local resection at the level of the pancreatic tail, left adrenal gland, left diaphragmatic pillar and ligamentum triangulare of the liver contiguous with the primary tumor), 1 had visceral pleural (left lower lobe wedge resection), and 1 had pancreatic metastases (resection of the pancreatic tail with en-bloc splenectomy). The 2 excluded patients were one with widespread peritoneal carcinomatosis of the upper abdomen who underwent complete peritoneal resection during esophageal resection, and one with bilateral pleural metastases and left lung metastases in whom palliative esophageal resection was performed with incomplete metastasectomy. Fourteen of 50 patients with disease progression discovered during restaging after neoadjuvant therapy or during an explorative procedure were oligometastatic; 10 (group 2) underwent maximal medical treatment. Five patients had liver metastases, 3 had brain metastases, and 2 had lung metastases. Of the 3 patients with brain metastases, 2 were treated with stereotactic radiotherapy and 1 with whole brain radiotherapy. The 4 excluded patients did not receive maximal medical therapy. Patient-related, disease-related and treatment-related characteristics of groups 1 and 2 are summarized in Table 1. There were

Table I. Patient, tumor, and treatment characteristics in groups $\mathrm{I}$ and 2.

\begin{tabular}{|c|c|c|}
\hline Variable & $\begin{array}{l}\text { Group I } \\
(n=10)\end{array}$ & $\begin{array}{l}\text { Group } 2 \\
(n=10)\end{array}$ \\
\hline $\begin{array}{l}\text { Median age (years) } \\
\text { [range] }\end{array}$ & 65 [44-73] & $67[44-77]$ \\
\hline Male & 8 & 8 \\
\hline Female & 2 & 2 \\
\hline $\begin{array}{l}\text { Median BMI }\left(\mathrm{kg} \cdot \mathrm{m}^{-2}\right) \\
\quad \text { [range] }\end{array}$ & $28.1(22.0-38.6)$ & $26.0(24.2-32.3)$ \\
\hline \multicolumn{3}{|l|}{ ASA physical status } \\
\hline I & $\mathrm{I}$ & 2 \\
\hline II & 6 & 8 \\
\hline III & 3 & 0 \\
\hline \multicolumn{3}{|c|}{ Charlson comorbidity index } \\
\hline 0 & 6 & 7 \\
\hline I-II & 4 & $\mathrm{I}$ \\
\hline III-IV & 0 & 2 \\
\hline$\geqslant \mathrm{V}$ & 0 & 0 \\
\hline \multicolumn{3}{|l|}{ Histology } \\
\hline Adenocarcinoma & 8 & 8 \\
\hline $\begin{array}{l}\text { Squamous cell } \\
\text { carcinoma }\end{array}$ & 2 & 2 \\
\hline \multicolumn{3}{|l|}{ Tumor location } \\
\hline Upper third & 0 & 0 \\
\hline Middle third & $\mathrm{I}$ & 2 \\
\hline Lower third & 5 & 5 \\
\hline $\begin{array}{l}\text { Gastroesophageal } \\
\text { junction }\end{array}$ & 4 & 3 \\
\hline \multicolumn{3}{|l|}{ Type of pretreatment } \\
\hline $\begin{array}{l}\text { Neoadjuvant } \\
\text { chemotherapy }\end{array}$ & 2 & 2 \\
\hline $\begin{array}{l}\text { Neoadjuvant } \\
\text { chemoradiotherapy }\end{array}$ & 8 & 8 \\
\hline \multicolumn{3}{|l|}{ Type of progression } \\
\hline Peritoneal & 1 & 0 \\
\hline Lung & 5 & 2 \\
\hline Pleura & 1 & 0 \\
\hline Brain & 0 & 3 \\
\hline Adrenal & 2 & 0 \\
\hline Pancreas & $\mathrm{I}$ & 0 \\
\hline Liver & 0 & 5 \\
\hline
\end{tabular}

ASA: American Society of Anesthesiologists; BMI: body mass index. 
no statistically significant differences between the 2 groups. Two hundred and twenty-eight of 552 patients in whom esophagectomy was performed with curative intent had at least one persistent pathological lymph node in the resected specimen (group 3). Median overall survival was 21.4 months in group 1, 12.1 months in group 2 and 20.2 months in group 3. Three-year survival was $26.7 \%, 10.0 \%$ and $32.1 \%$ respectively. Overall survival was significantly longer in group 1 compared to group $2(p=0.042)$. There was no difference in overall survival between group 1 and group $3(p=0.88)$, but overall survival in group 3 was significantly longer compared to group 2 ( $p=0.002$; Figure 1).

\section{Discussion}

This study shows that in locally advanced esophageal cancer patients undergoing multimodal treatment with curative intent, those with unexpected oligometastatic disease have almost double the median overall survival when esophagectomy and concurrent metastasectomy is performed, compared to patients in a comparable disease stage who have maximal medical treatment. This is in line with the very recent results of the AIO-FLOT3 trial in limited metastatic gastric and junctional carcinoma, which also confirmed a favorable outcome in patients who proceeded to surgery. ${ }^{14}$ Locally advanced esophageal cancer patients treated with curative intent have a 5-year overall survival rate of only $33 \%$ according to the Worldwide Esophageal Cancer Collaboration database; the majority die from recurrent disease within
24 months. ${ }^{15}$ This is in spite of thorough clinical staging to exclude distant metastases, indications for multimodal treatment, and restaging to exclude interval metastases after neoadjuvant treatment in most cases. Other carcinoma types, such as breast cancer, colorectal cancer, and non-small-cell lung cancer have better prognoses in patients treated with curative intent, and this might be one of the reasons why protocols aiming at treatment of synchronous oligometastatic diseases have been successful in these tumor types. ${ }^{9-11}$ On the other hand, selected patients with metachronous oligometastatic esophageal cancer (single distant organ recurrent disease) are known to benefit more from surgical therapy than medical therapy alone. ${ }^{16,17}$

The primary objective of this study was not to justify the indication for surgery in oligometastatic disease at restaging in patients with esophageal carcinoma but to answer the practical question of whether one has to abort the surgical resection in cases of unexpected oligometastatic disease regardless of meticulous clinical staging. Erhunmwunsee and colleagues ${ }^{18}$ investigated the outcome of esophageal resection in patients with suspicious lesions on staging imaging, which disappeared on restaging after neoadjuvant therapy. They found no difference in survival of patients with or without suspicious lesions, suggesting giving them the benefit of doubt; but when metastatic disease was confirmed at resection, none of these patients survived more than 2.5 years. However, the authors did not mention whether the metastases were resected. Esophagectomy in $\mathrm{yN}+$ patients is never questioned because pathological lymph nodes cannot yet be

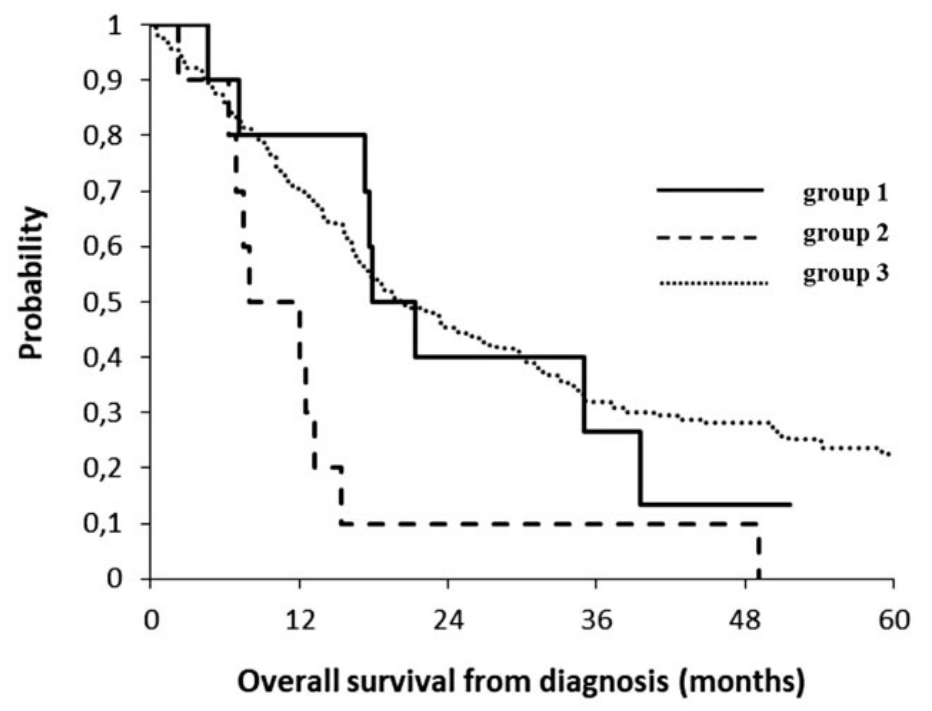

\begin{tabular}{|l|c|c|c|c|c|c|}
\hline Group1 & 10 & 9 & 5 & 2 & 2 & 1 \\
\hline Group 2 & 10 & 6 & 1 & 1 & 1 & 0 \\
\hline Group 3 & 228 & 161 & 89 & 64 & 39 & 27 \\
\hline
\end{tabular}

Figure 2. Overall survival of surgically treated patients with unexpected oligometastatic disease (group I), medically treated patients with oligometastatic disease (group 2) and surgically treated patients with persistent pathological lymph nodes (group 3 ). 
adequately predicted by clinical restaging, ${ }^{19}$ and surgery is the only chance of cure. Even in patients who are medically fit, locoregional $\mathrm{ycN}+$ is not seen as a contraindication for surgery. Therefore, we compared both oligometastatic groups with a third group of persistent proven pathological lymph nodes after neoadjuvant treatment. Our data confirm that overall survival in esophageal cancer patients with unexpected synchronous oligometastatic disease at the time of surgery following neoadjuvant therapy is similar to that in patients with persistent pathological lymph nodes. The fact that some patients with synchronous metastatic disease have a better prognosis than others with only lymph node metastases is probably due to differences in tumor biology. ${ }^{16}$ In the near future, circulating biomarkers will probably be useful in selecting patients with lymph node dissemination or distant spread for radical treatment. ${ }^{20}$

Potential weaknesses of this study are obviously the retrospective design and the low numbers of patients included in groups 1 and 2. Furthermore, although efforts were made to obtain comparable groups in terms of extent of metastatic disease in groups 1 and 2 (single-organ oligometastatic with maximal surgical versus medical therapy), one could argue that patients with liver metastases and brain metastases have less favorable outcomes than those with adrenal and lung metastases. However, most evidence is based on handling metachronous rather than synchronous disease ${ }^{17,18}$

In conclusion, overall survival in patients undergoing radical surgery in unexpected single-organ oligometastatic disease during esophagectomy is longer compared to medically treated oligometastatic patients. Furthermore, their overall survival is comparable to that of patients with persistent pathological lymph nodes. Therefore, palliative resection in unexpected oligometastatic disease seems to be justified.

Presented at the 26th ASCVTS Meeting, Winner of AATS Foundation Award Session, Moscow, May 262018.

\section{Declaration of conflicting interests}

The author(s) declared no potential conflicts of interest with respect to the research, authorship, and/or publication of this article.

\section{Funding}

The author(s) disclosed receipt of the following financial support for the research, authorship, and/or publication of this article: Lieven Depypere was supported by the Clinical Research Fund of the University Hospitals Leuven. This research received no specific grant from any funding agency in the public, commercial, or not-for-profit sectors.

\section{ORCID iD}

Lieven P Depypere (D) http://orcid.org/0000-0001-8230-5649 Hans Van Veer (D http://orcid.org/0000-0003-1153-8298

\section{References}

1. Lordick F, Mariette C, Haustermans K, Obermannová R and Arnold D; ESMO Guidelines Committee. Oesophageal cancer: ESMO Clinical Practice Guidelines for diagnosis, treatment and follow-up. Ann Oncol 2016; 27: v50-57.

2. Ajani JA, D'Amico TA, Almhanna K, et al. Esophageal and esophagogastric junction cancers, version 1.2015. J Natl Compr Canc Netw 2015; 13: 194-227.

3. Shapiro J, van Lanschot JJ, Hulshof MC, et al. Neoadjuvant chemoradiotherapy plus surgery versus surgery alone for oesophageal or junctional cancer (CROSS): long-term results of a randomised controlled trial. Lancet Oncol 2015; 16: 1090-1098.

4. Kelsen DP, Winter KA, Gunderson LL, et al. Long-term results of RTOG trial 8911 (USA Intergroup 113): a random assignment trial comparison of chemotherapy followed by surgery compared with surgery alone for esophageal cancer. J Clin Oncol 2007; 25: 3719-3725.

5. Hulshoff JB, Smit JK, van der Jagt EJ and Plukker JT. Evaluation of progression prior to surgery after neoadjuvant chemoradiotherapy with computed tomography in esophageal cancer patients. Am J Surg 2014; 208: 73-79.

6. Bruzzi JF, Swisher SG, Truong MT, et al. Detection of interval distant metastases: clinical utility of integrated CT-PET imaging in patients with esophageal carcinoma after neoadjuvant therapy. Cancer 2007; 109: 125-134.

7. Blom RL, Schreurs WM, Belgers HJ, et al. The value of post-neoadjuvant therapy PET-CT in the detection of interval metastases in esophageal carcinoma. Eur J Surg Oncol 2011; 37: 774-778.

8. Anderegg MC, de Groof EJ, Gisbertz SS, et al. 18F-FDG PET-CT after neoadjuvant chemoradiotherapy in esophageal cancer patients to optimize surgical decision making. PLoS One 2015; 10: e0133690.

9. Trovo M, Furlan C, Polesel J, et al. Radical radiation therapy for oligometastatic breast cancer: results of a prospective phase II trial. Radiother Oncol 2018; 126: 177-180.

10. Kwint M, Walraven I, Burgers S, et al. Outcome of radical local treatment of non-small cell lung cancer patients with synchronous oligometastases. Lung Cancer 2017; 112: 134-139.

11. Aigner F, Pratschke $\mathbf{J}$ and Schmelzle M. Oligometastatic disease in colorectal cancer-how to proceed? Visc Med 2017; 33: 23-28.

12. Nafteux P, Depypere L, Van Veer H, Coosemans W and Lerut T. Principles of esophageal cancer surgery, including surgical approaches and optimal node dissection (2- vs. 3-field). Ann Cardiothorac Surg 2017; 6: 152-158.

13. Li D, Zhu X, Wang H, Qiu M and Li N. Should aggressive thoracic therapy be performed in patients with synchronous oligometastatic non-small cell lung cancer? A meta-analysis. J Thorac Dis 2017; 9: 310-317.

14. Al-Batran SE, Homann N, Pauligk C, et al. Effect of neoadjuvant chemotherapy followed by surgical resection on survival in patients with limited metastatic gastric or gastroesophageal junction cancer: the AIO-FLOT3 Trial. JAMA Oncol 2017; 3: 1237-1244. 
15. Rice TW, Lerut TE, Orringer MB, et al. Worldwide Esophageal Cancer Collaboration: neoadjuvant pathologic staging data. Dis Esophagus 2016; 29: 715-723.

16. Depypere L, Lerut T, Moons J, et al. Isolated local recurrence or solitary solid organ metastasis after esophagectomy for cancer is not the end of the road. Dis Esophagus 2017; 30: 1-8.

17. Schizas D, Lazaridis II, Moris D, et al. The role of surgical treatment in isolated organ recurrence of esophageal cancer-a systematic review of the literature. World J Surg Oncol 2018; 16: 55.

18. Erhunmwunsee L, Englum BR, Onaitis MW, et al. Impact of pretreatment imaging on survival of esophagectomy after induction therapy for esophageal cancer: who should be given the benefit of the doubt? Esophagectomy outcomes of patients with suspicious metastatic lesions. Ann Surg Oncol 2015; 22: 1020-1025.

19. Ngamruengphong S, Sharma VK, Nguyen B and Das A. Assessment of response to neoadjuvant therapy in esophageal cancer: an updated systematic review of diagnostic accuracy of endoscopic ultrasonography and fluorodeoxyglucose positron emission tomography. Dis Esophagus 2010; 23: 216-231.

20. Chiapponi C, Berlth F, Plum PS, et al. Oligometastatic disease in upper gastrointestinal cancer - how to proceed? Visc Med 2017; 33: 31-34. 